\title{
DISCRETE AND DIFFERENTIAL EQUATIONS IN APPLIED MATHEMATICS
}

In the contribution we map investigations performed in mathematics at the Faculty of Science in 2003-2007. Main directions developed at the faculty are described and some of the latest achievements are presented. We illustrate some results from control theory for discrete delayed equations, perturbed linear problems for discrete equations, results concerning the structure of solutions of delayed differential equations, results of orthogonal polynomials, and a description of some numerical investigations in discrete least-squares and collocation methods for numerical solution of a boundary value and eigenvalue problems. Some of accompanying mathematical activities are mentioned as well.

\section{Introductory Words about Mathematics at the Faculty}

The last five years are distinguished by huge research in various mathematical disciplines in mathematical departments of the faculty. This research was also stimulated by intensive mathematical collaboration with mathematicians from outside and with participation of our colleagues in international conferences. We also organized several international meetings. We mention, at least, two successful meetings - the IMC conference (International Mathematical Conference) in 2003 in Zilina devoted to the $50^{\text {th }}$ anniversary of Zilina University and 5th anniversary of the Faculty of Science, and the ICDDEA 2006 conference (International Conference on Differential and Difference Equations and Application) in Rajecke Teplice. The mathematical research in this period was supported by eight scientific projects VEGA (the Grant Agency of the Slovak Republic) as well as by two international scientific projects and three projects KEGA (the Cultural and Educational Grant Agency of the Slovak Republic). Recently, 6 members of the staff successfully defended their Ph.D. theses in applied mathematics and at present, two members of the staff are preparing for their habilitation and professor procedures in mathematics.

One of the traditional direction of research is the field of functional differential equations, difference and discrete equations and their applications. This tradition goes back to such outstanding authorities as prof. Pavol Marusiak (1935-2000) and prof. Jozef Moravcik (1934-2005). Another traditional direction of research is the field of orthogonal polynomials which was founded by prof. Josef Korous (1906-1981).

\section{Main Mathematical Trends}

We will give a short description of main mathematical directions pursued at the faculty together with formulations of selected important results.

\subsection{Difference Equations}

\subsubsection{Controllability of Linear Discrete Equations}

One of rapidly developed directions is investigation of controllability of linear discrete systems with delay. Such research is important due to numerous applications of mathematical models in natural sciences, economics and engineering. Let us consider a discrete system with pure delay

$$
\Delta x(k)=B x(k-m)+b u(k),
$$

where $m \geq 1$ is a fixed integer, $k \in Z_{0}^{\infty}, Z_{s}^{q}:=\{s, s+1, \ldots, q\}$, $B$ is a constant $n \times n$ matrix,

$$
\Delta x(k)=x(k+1)-x(k),
$$

$x: Z_{-m}^{\infty} \rightarrow R^{n}$ is unknown solution, $b \in R^{n}$ is a given nonzero vector and $u: Z_{0}^{\infty} \rightarrow R$ is the input scalar function. Together with (1) we consider an initial (Cauchy) problem

$$
x(k)=\varphi(k)
$$

\footnotetext{
* Miroslava Ruzickova

Department of Mathematical Analysis and Applied Mathematics, Faculty of Science, Universtiy of Zilina, Slovakia,

E-mail: miroslava.ruzickova@fpv.uniza.sk
} 
with a given initial function $\varphi: Z_{-m}^{0} \rightarrow R^{n}$.

System (1) is called relatively controllable, if for any initial function $\varphi: Z_{-m}^{0} \rightarrow R^{n}$, any finite terminal state $x=x^{*} \in R^{n}$, and any finite terminal point $k_{1}$ equal or greater than a fixed integer $k^{*} \in Z_{1}^{\infty}$ there exists a discrete function $u^{*}: Z_{0}^{k_{1}-1} \rightarrow R$ such that the system (1) with the input $u=u^{*}$ has a solution $x^{*}: Z_{-m}^{k_{1}} \rightarrow R^{n}$ such that $x^{*}\left(k_{1}\right)=x^{*}$ and $x^{*}(k)=\varphi(k)$ if $k \in Z_{-m}^{0}$.

The crucial role is, in several investigations of this type, played by so called discrete matrix delayed exponential (see [8]) defined as follows:

$$
e_{m}^{B k} \stackrel{\text { def }}{=}\left\{\begin{array}{l}
\Theta \text { if } k \in Z_{-\infty}^{-m-1}, \\
I \text { if } k \in Z_{-m}^{0}, \\
I+B \cdot\left(\begin{array}{l}
k \\
1
\end{array}\right) \text { if } k \in Z_{1}^{m+1}, \\
\ldots+B \cdot\left(\begin{array}{l}
k \\
1
\end{array}\right)+B^{2} \cdot\left(\begin{array}{c}
k-m \\
2
\end{array}\right) \\
+\ldots+B^{\ell} \cdot\left(\begin{array}{c}
k-(\ell-1) m \\
\ell
\end{array}\right) \\
\text { if } k \in Z_{(\ell-1)(m+1)+1}^{((m+1)} \\
\ell=0,1,2, \ldots
\end{array}\right.
$$

where $\Theta$ is $n \times n$ null matrix.

The discrete matrix delayed exponential can be successfully used for representing the solutions of discrete systems. In the case of the problem (1), (2) we have:

$$
\begin{gathered}
x(k)=e_{m}^{B K} \varphi(-m)+\sum_{j=-m+1}^{0} e_{m}^{B(k-m-j)} \Delta \varphi(j-1) \\
+\sum_{j=1}^{k} e_{m}^{B(k-m-j)} b u(j-1)
\end{gathered}
$$

where $k \in Z_{1}^{\infty}$.

Let us define an auxiliary $n \times n$ matrix

$$
S \stackrel{\text { def }}{=}\left(b, B b, B^{2} b, \ldots, B^{n+1} b\right)
$$

and the vector

$$
\xi \stackrel{\text { def }}{=} x^{*}-e_{m}^{B k_{1}} \varphi(-m)-\sum_{j=-m+1}^{0} e_{m}^{B\left(k_{1}-m-j\right)} \Delta \varphi(j-1)
$$

In [9] there are given conditions for relative controllability and the control function $u=u^{*}(k), k \in Z_{0}^{k_{1}-1}$ is found for the problem

$$
\begin{aligned}
& \Delta x(k)=B x(k-m)+b u(k), k \in Z_{0}^{k_{1}-1}, \\
& x(k)=\varphi(k), k \in Z_{-m}^{0},
\end{aligned}
$$

$$
x\left(k_{1}\right)=x^{*} .
$$

Theorem 1 Problem (3)-(5) is relatively controllable if and only if assumptions

$$
\text { rank } S=n
$$

and

$$
k_{1} \geq(n-1)(m+1)+1
$$

hold simultaneously

Theorem 2 Let the conditions of relative controllability (6), (7) be valid. Then a control function $u=u^{*}$ for the problem (3)-(5) can be expressed in the form

$$
u^{*}(k)=b^{T}\left(e_{m}^{B\left(k_{1}-m-k-1\right)}\right)^{T} G^{-1} \xi
$$

where the matrix

$$
G \stackrel{\text { def }}{=} \sum_{j=1}^{k_{1}} e_{m}^{B\left(k_{1}-m-j\right)} b b^{T}\left(e_{m}^{B\left(k_{1}-m-j\right)}\right)^{T}
$$

is nonsingular.

\subsubsection{Perturbed Linear Problems, Asymptotic Decomposition of their Solutions}

Let us consider the perturbed non-homogeneous linear problem in the form

$$
x(k+1)=A(k) x(k)+f(k)+\varepsilon A_{1}(k) x(k)
$$

where $A, A_{1}, f \in B(Z), B(Z)$ is the Banach space of vector-valued functions $x: Z \rightarrow R^{n}$ bounded on $Z$ with the $\|x\|=\sup _{k \in Z}|x(k)|$, $|x(k)|:=\|x\|_{R^{n}}$ and $Z$ denotes the set of all integers. Let us assume that the generating problem

$$
x(k+1)=A(k) x(k)+f(k)
$$

has no solutions bounded on the whole line $Z$ for arbitrary $f \in B(Z)$ and system

$$
x(k+1)=A(k) x(k)
$$

has a dichotomy on $Z_{+}$and $Z_{-}$. Here symbols $Z_{+}$or $Z_{-}$denote the sets of all non-negative or non-positive integers.

A typical solved problem is: Is it possible to make the problem (9) solvable by means of linear perturbations and, if it is possible, then what kind of the perturbation $A_{1}$ should be for the problem (8) to become solvable everywhere?

This question can be answered with the help of $d \times r$ matrix

$$
B_{0}=\sum_{k=-\infty}^{+\infty} H_{d}^{*}(k+1) A_{1}(k) X_{T}(k),
$$


the construction of which involves the perturbation term of problem (8). Using the method of generalized inverse operators [6] we can find bifurcation conditions, when solutions bounded on the whole line $Z$ of problem (8) appear in the form of a part of Laurent series in powers of a small parameter $\varepsilon$. Results obtained can be illustrated by one theorem from [5]

Theorem 3 Consider the problem (8) and assume that for arbitrary $f \in B(Z)$ generating problem (9) has no solutions bounded on $Z$ and (10) has a dichotomy on $Z_{+}$and $Z_{-}$with projectors $P$ and $Q$ respectively. If rank $B_{0}=d$ then, for sufficiently small $\varepsilon \in\left(0, \varepsilon_{0}\right]$ :

1) The operator $L_{\varepsilon}: B(Z) \rightarrow B(Z)$

$\left(L_{\varepsilon} x\right)(k) \stackrel{\text { def }}{=} z(k+1)-A(k) x(k)-\varepsilon A_{1}(k) x(k)$

is a Fredholm one with

$\operatorname{ind} L_{\varepsilon}=\operatorname{dimker} L_{\varepsilon}-\operatorname{dimker} L_{\varepsilon}^{*}=\rho=r-d$

where the operator $L_{\varepsilon}^{*}$ is the adjoint one to $L_{\varepsilon}$

$\left(\right.$ ind $\left.L_{0}=\rho=r-d, \operatorname{dim} \operatorname{ker} L_{0}=r, \operatorname{dimker} L_{0}^{*}=d\right)$.

2) The homogeneous problem (10) has a $\rho$-parametric family of solutions

$x_{0}\left(k, \varepsilon, c_{\rho}\right)=\sum_{i=-1}^{\infty} \varepsilon^{i} \bar{X}_{i}(k) P_{B_{\rho} C_{\rho}}$

where $c_{\rho} \in R^{\circ}$ is arbitrary and $\rho=\operatorname{dim}$ ker $E_{\varepsilon}$ with the properties:

$x_{0}\left(\cdot, \varepsilon, c_{\rho}\right) \in B(Z), x_{0}\left(k, \cdot, c_{\rho}\right) \in C\left(0, \varepsilon_{0}\right]$.

The terms $\bar{X}_{i}(k)$ can be determined.

3) The problem adjoint to (11) has only trivial bounded solution

$\left(\operatorname{dim} \operatorname{ker} L_{\varepsilon}^{*}=0, \varepsilon \in C\left(0, \varepsilon_{0}\right]\right)$.

4) The problem (8) has, for arbitrary $f \in B(Z)$, a $\rho$-parametric set of solutions

$x(k, \varepsilon)=z\left(k, \varepsilon, c_{\rho}\right): x\left(\cdot, \varepsilon, c_{\rho}\right) \in B(Z)$,

$x\left(k, \cdot, c_{\rho}\right) \in C\left(0, \varepsilon_{0}\right]$

in the form of the series

$x\left(k, \varepsilon, c_{\rho}\right)=\sum_{i=-1}^{\infty} \varepsilon^{i}\left[\bar{x}_{i}\left(k, \bar{c}_{i}\right)+\bar{X}_{i}(k) P_{B_{\rho} c_{\rho}}\right]$

convergent for sufficiently small $\varepsilon \in\left(0, \varepsilon_{0}\right]$; where

$\bar{x}_{i}\left(k, \bar{c}_{i}\right), \bar{c}_{i}$ and $\bar{X}_{i}(k)$

can be determined.
Similarly, conditions for the existence of solutions bounded on the whole line $Z$, which turn into one of generating solutions of system (8), for nonlinear difference system

$$
x(k+1)=A(k) x(k)+f(k)+\varepsilon Z(x, k, \varepsilon)
$$

were derived. The proposed approach to the analysis of boundaryvalue problems for systems of ordinary differential equations can be also applied (with relevant modifications) to systems with delayed argument [3] and to finding conditions for the bifurcation of a weak $T$-periodic solution of the linear abstract wave equation and suggest an algorithm for constructing of such solution [4]. Weak solutions were studied and the Vishik-Lyusternik method has permitted one to find the desired weak $T$-periodic solution in the form of part of the Laurent series. Boundary value problems for systems of second order linear and nonlinear ordinary differential equations and linear impulsive systems are studied using the Vishik-Lyusternik method and the theory of pseudoinverse matrices (in the sense of Moore-Penrose) in [28, 29].

\subsubsection{Bounded Solutions}

A lot of effort was devoted to investigation of existence of bounded solutions of different solutions via different methods that are used in the previous part. With so called retract type technique and Lyapunov technique, new methods and results were published, e.g., in [14, 20, 21, 35]. Part of results obtained was extended to some special and arbitrary time-scales.

\subsection{Differential Equations}

\subsubsection{Oscillatory and Asymptotic Properties of Solutions, Periodic Solutions, Stability of Solutions}

The subject of research was the investigation of oscillatory and asymptotic properties of nonlinear functional differential equations and systems with retarded arguments [22, 23, 31]-[33]. For example, a nonlinear delay differential equation of the form

$$
\dot{x}(t)+p(t) x(t)=q(t) f(x(\tau(t)))
$$

was investigated for $t \geq t_{0}$, where

$$
p, q:\left[t_{0}, \infty\right) \rightarrow R, f: R \rightarrow R
$$

are continuous functions and $\tau:\left[t_{0}, \infty\right) \rightarrow(0, \infty)$ is a continuous increasing function, $\lim \tau(t)=\infty$. Together with (12) we consider an initial problem

$$
x(t)=\varphi(t), t \in\left[t_{-1}, t_{0}\right], t_{-1}=\tau\left(t_{0}\right)
$$

with a continuous initial function $\varphi$. Let us denote the solution of (12), (13) as $x_{\varphi}(t)$. The next stability theorem illustrates the character of results obtained. 
Theorem 4 Suppose that an interval $I=[0, b], b>0$, is mapped by finto itself, $\left(\left[t_{-1}, t_{0}\right], I\right), 0<q(t) \leq p(t)$ for $t \in\left[t_{0}, \infty\right)$,

$$
\int_{t_{0}}^{\infty} p(s) d s=\infty
$$

and

$$
\lim _{t \rightarrow \infty} \int_{t_{0}}^{t} q(s) \exp \left(-\int_{s}^{t} p(u) d u\right) d s=0 .
$$

Then $\lim _{t \rightarrow \infty} x_{\varphi}(t)=0$.

The cognate equations have recently found a variety of applications in several fields of natural sciences. Especially the nonlinear differential equations with time delays model processes in radiophysics, optics, neural interactions, etc. This is due to influence of the past history of the processes on their evolution.

Research was also focused on the behavior of linear or nonlinear integro-differential equations with distributed delays or discrete delays [1, 2]. Such equations recently appeared in the theory of circulating fuel nuclear reactor and also can be a good model in one dimensional viscoelasticity.

In other articles some economic models are treated (e.g. [24]). For example, the stability of neoclassical growth model or the dynamics of basic macroeconomical model of national income is observed.

At the present time we are interested in existence of positive periodic solutions of functional differential equations. Periodic processes can occur in some population models [25].

In applications it is important to know if all solutions of given equation are for $t \rightarrow+\infty$ convergent to a finite limit or if there are divergent solutions. Such problems are solved in [16, 18] for equations of the form

$$
\dot{y}(t)=\beta(t)[y(t-\delta)-y(t-\tau)]
$$

with arbitrary positive and continuous function $\beta$ and with positive constants $\delta, \tau, \tau>\delta$. New results on existence of solutions of singular initial problems of systems of differential equations were derived. The main results are given in [17, 19].

\subsubsection{Structure of Solutions, Existence of Positive Solutions}

As was noted above, equations with time lag are used in many mathematical models of different phenomena. A general qualitative result about the structure of solutions of linear differential equations with delay was proved in [15]. Let us consider a linear equation

$$
\dot{x}(t)=-\sum_{i=1}^{m} c_{i}(t) x\left(t-r_{i}(t)\right)
$$

with $c_{i}:\left[t^{*}, \infty\right) \rightarrow R, i=1, \ldots, m$ and $r_{i}:\left[t^{*}, \infty\right) \rightarrow(0, r]$

Theorem 5 Let us suppose the existence of a positive solution $\tilde{x}$ of (14) on $\left[t^{*}-r, \infty\right)$. Then either every solution $x$ of $(14)$ on $\left[t^{*}-r, \infty\right)$ is represented in a unique way by the formula

$$
x(t)=\tilde{x}(t)(K+\varphi(t)),
$$

where $K \in R$ depends on $x$, and $\varphi$ is a continuous function dependent on $x$ and satisfying $\varphi(+\infty)=0$, or every solution $x$ of (14) on $\left.\mid t^{*}-r, \infty\right)$ is represented in a unique way by the formula

$$
x(t)=\tilde{x}(t)(K Y(t)+\delta(t)),
$$

where $Y$ is a continuous, increasing function which is the same for each $x$, satisfies $Y(+\infty)=\infty, K \in R$ depends on $x$, and $\delta$ is a bounded continuous function dependent on $x$.

Theorem 6 Suppose the existence of a positive solution of (14) on $\left[t^{*}-r, \infty\right)$. Then there exist two positive solutions $x_{1}$ and $x_{2}$ of $(14)$ on $\left[t^{*}-r, \infty\right)$ satisfying the relation

$$
\lim _{t \rightarrow \infty} \frac{x_{2}(t)}{x 1(t)}=0
$$

such that every solution $x=x(t)$ of $(14)$ on $\left.\mid t^{*}-r, \infty\right)$ can be represented by the formula

$$
x(t)=K x_{1}(t)+O\left(x_{2}(t)\right),
$$

where the constant $K$ depends on $x$.

In [13] (see [10]-[12] as well) a linear equation

$$
\dot{x}=-a(t) x(t-r)
$$

being a particular case of (14) is considered, where $a:\left[t_{0}, \infty\right) \rightarrow$ $\rightarrow R^{+}=(0, \infty)$ and $\mathrm{r} \in R^{+}$and assume $0<A \leq a(t) \leq B<$

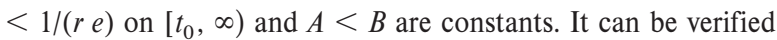
that the equation for $\lambda: \lambda=A e^{\lambda r}$ has just two different positive roots $\lambda_{A}^{*}, \lambda_{A}^{* *}, \lambda_{A}^{*}<\lambda_{A}^{* *}$ and the equation $\lambda: \lambda=>B e^{\lambda r}$ has just two different positive roots $\lambda_{B}^{*}, \lambda_{B}^{* *}, \lambda_{B}^{*}<\lambda_{B}^{* * *}$ such that $\lambda_{A}^{*}<\lambda_{B}^{*}$ and $\lambda_{A}^{* *}>\lambda_{B}^{* *}$. Under some additional assumptions it is proved that there are positive solutions $x=x_{1}$ and $x=x_{2}$ of (17) such that

$$
\begin{aligned}
& e^{-\lambda_{B}^{*}\left(t-t_{0}+r\right)} \leq x_{1}(t) \leq e^{-\lambda_{A}^{*}\left(t-t_{0}+r\right)}, \\
& e^{-\lambda_{A}^{*+t}} t^{p}<x_{2}(t) \leq e^{-\lambda_{B}^{*+t}} t^{q}
\end{aligned}
$$

where $p<0$ and $q>0$ are fixed numbers. Solutions $x_{1}(t), x_{2}(t)$ satisfy (15) and every solution of (17) can be represented by formula (16). A part of results remain valid for a general functional equation $\dot{x}(t)=-f\left(t, x_{t}\right)$ under the main assumption

$$
A \varphi(-r) \leq f(t, \varphi) \leq B \varphi(-r) .
$$




\subsection{Numerical Mathematics}

In the latest period a deep research concerning some numerical aspects in discrete least-squares and collocation methods for numerical solution of boundary value and eigenvalue problems were performed.

Although the least-squares and collocation methods belong to the classical tools in the numerical solution of partial differential equations, there is still enough space for their investigation with respect to efficiency, stability and applicability in important realworld problems. During the years of research and numerical experiments the scientists and engineers have revealed that the crucial point of successful application of the mentioned methods is based on the optimal choice of matching points ensuring the highest possible accuracy allowed by the used trial functions and as small as possible condition number of the resulting coefficient matrices.

One of the possible sets of suitable matching points could be taken the Lebesgue points which are optimal interpolation points minimizing the Lebesgue constant $\Lambda_{n}$. This constant plays central role in the Lagrange interpolation process according to the inequality

$$
\left\|u-L_{n}\right\| \leq\left(1+\Lambda_{n}\right)\left\|u-p_{n}\right\|,
$$

where $L_{n}$ is the Lagrange interpolation polynomial of the function $u$, i.e., $L_{n}\left(x_{i}\right)=u\left(x_{i}\right), i=0,1,2, \ldots, n$ and $p_{n}$ is the best polynomial approximation of $u$. Unfortunately, as written in [34]: "Almost nothing seems to be known about Lebesque points in more than one dimension. Nor are we aware of a feasible method for computing them numerically."

Another set of suitable matching points can be considered the Fekete points for given domain which maximize the corresponding Vandermonde determinant. These points are known for rectangular and triangular domains, however, the computation of the Fekete points for general two-dimensional domains with complicated boundary shape or multiple connected domains is still an open problem. To avoid this terra incognita we concentrated our attention to the most important requirement in the matrix computations expressed in the following inequality

$$
\frac{\left\|x-x_{a}\right\|}{\|x\|} \leq C \cdot \operatorname{cond}(A) \times\left(\frac{\|\Delta A\|}{\|A\|}+\frac{\|\Delta b\|}{\|b\|}\right) .
$$

This inequality describes how much the roundoff error propagation may destroy accuracy of the approximate solution $x_{a}$ of the system of linear equation $A x=b$ using LU-decomposition. It is evident that small condition number $(\operatorname{cond}(A))$ of the coefficient matrix $A$ guarantees small error of the approximate solution $x_{a}$. Consequently, this dependence can be used to reveal a new way of computing another set of points which could be suitable for our purposes. As a new possibility we tried to find suitable matching points which minimize condition number of the coefficient matrix $A$. The results concerning discrete least-squares solution of the Poisson problem

$$
-\Delta u(x)=f(x), \quad x \in \Omega
$$

and the eigenvalue problem

$$
-\Delta u(x)=\lambda g(x) u(x), \quad x \in \Omega
$$

subject to the homogeneous Dirichlet boundary conditions

$$
u(x)=0, \quad x \in \partial \Omega
$$

for rectangular, circular and triangular domain $\Omega$ are presented $[36,37]$. The eigenvalue approximations computed for these domains show that at least 10 digits of accuracy for the first 10 eigenvalues may be obtained using less than 200 basis functions. Moreover, the conformal mapping technique can be used for computing the eigenvalue approximations for domains with exotic shapes generated by conformal mapping of rectangular, circular and triangular domains.

\subsection{Orthogonal Polynomials and their Applications}

In [30] several theorems are proved expressing integrals involving polynomials orthogonal in a finite symmetric interval by means of their coefficients. In the proofs for the polynomials $\left\{P_{n}(x)\right\}_{n=0}^{\infty}$ orthonormal in a finite symmetric interval I associated with even weight function $v(x)$ it is used the well-known following property: $P_{n}(-x)=(-1)^{n} P_{n}(x)$. Denote

$$
P_{n}(x)=\sum_{k=0}^{n} p_{k}^{(n)} x^{n-k}, \quad p_{0}^{(n)}>0
$$

and

$$
p_{0}=0, \quad p_{n}=\frac{p_{0}^{(n-1)}}{p_{0}^{(n)}}
$$

for natural $n$. The following results are valid:

$$
\begin{aligned}
& \int_{I} x^{2} P_{n}^{2}(x) v(x) d x=p_{n+1}^{2}+p_{n}^{2}, \\
& \int_{I} x^{3} P_{n}(x) P_{n-1}(x) v(x) d x=p_{n}\left(p_{n+1}^{2}+p_{n}^{2}+p_{n-1}^{2}\right),
\end{aligned}
$$

and for a positive integer $k<n$ we proved:

$$
\int_{I} x^{k} P_{n}(x) P_{n-k}(x) v(x) d x=\prod_{i=0}^{k-1} p_{n-i} .
$$

In $[7]$ we studied the classical Jacobi orthogonal polynomials $\left\{P_{n}(x) ; \alpha, \beta\right\}_{n=0}^{\infty}$ which are orthogonal in the interval $I=[-1,1]$ with respect to the weight function

$$
J(x)=(1-x)^{\alpha}(1+x)^{\beta},
$$

where $\alpha>-1, \beta>-1$. Especially their special cases - ultraspherical polynomials (when $\alpha=\beta$ in $J(x)$ ), Chebyshev polynomials of the first kind ( $\alpha=\beta=-1 / 2$ in $J(x)$ ), Chebyshev polyno- mials of the second kind $(\alpha=\beta=1 / 2$ in $J(x))$ and Legendre polynomials $(\alpha=\beta=0$ in $J(x))$ are often used in the approximation theory 
and they appear as the solutions of some problems of mathematical physics. For certain integrals of classical Jacobi orthonormal polynomials there are derived formulas interconnecting them with cyclometric functions. It holds:

$$
\begin{aligned}
& \text { Theorem } 7 \text { For } x \in[-1,1] \\
& \qquad \int_{x}^{1} P_{n}^{2}(t ; \alpha, \beta) J(t) d t=\frac{1}{\pi} \arccos x+g_{2}^{(n)}(x) \\
& \text { and } \\
& \qquad \int_{x}^{1} t P_{n}^{2}(t ; \alpha, \beta) J(t) d t=\frac{1}{\pi} \sqrt{1-x^{2}}+g_{3}^{(n)}(x) \\
& \text { where for } i=2,3: x \in[-1,1] \Longrightarrow\left|g_{i}^{(n)}(x)\right|<c_{i} n^{-1} .
\end{aligned}
$$

Another view of our research on orthogonal polynomials could be taken through the works [26, 27] dealing with generalized function spaces. Especially the work [27] (cited 95 times in SCI) can be estimated as a pioneering work in this direction.

\section{Mathematical Perspectives}

The youngest faculty celebrates 10 years of its existence. Nevertheless, this short period showed that applied mathematics successfully developed in both national and international contexts and has a promising perspective in the future. Presently, the mathematical groups are supported by four grant projects of the Grant Agency of Slovak Republic (VEGA), one international project and several institutional projects. The international collaboration is large and results mainly in publishing common research papers. In spite of it we will try to upgrade these contacts and create international research groups supported by scientific grants. The forthcoming stimulating mathematical event, organized by the faculty will be the ICDDEA 2008 conference (International Conference on Differential and Difference Equations and Application) in Strecno.

\section{Acknowledgment}

Preparation of this survey was supported by the Grant No 1/3238/06 of the Grant Agency of Slovak Republic (VEGA).

\section{References}

[1] BACOVA, B., DOROCIAKOVA, B.: Oscillation of Nonlinear Differential Systems with Retarded Arguments, Czechoslovak Math. Journal 55 (2005), no. 1, 255-262.

[2] BACOVA, B., OLACH, R.: Oscillation of Nonlinear Delay Integro-differential Equations, Tatra Mount. Math. Publ., in press.

[3] BOICHUK, A.: Perturbed Predholm BVP's for Systems of Delay Differential Equations, Equadiff 2003, World Sci. Publ., Hackensack, NJ, 2005, 1033-1035.

[4] BOICHUK, A., KOROSTIL, I., FECKAN, M.: Bifurcation Conditions of Solutions for Abstract Wave Equations, Differ. Equ. 43 (2007), No. 4, 495-502 ; translation from Differ. Uravn. 43 (2007), no. 4, 481-487.

[5] BOICHUK, A., RUZICKOVA, M.: Solutions Bounded on the Whole Line for Perturbed Difference Systems, Elaydi, Saber (ed.) et al., Proc. of the $8^{\text {th }}$ International Conference on Difference Equations and Appli-cations, Masaryk University, Brno, Czech Republic, Boca Raton, FL: Chapman \& Hall/CRC. (2005), 51-59.

[6] BOICHUK, A., SAMOILENKO, M.: Generalized Inverse Operators and Fredholm Boundary Value Problems, VSP, Utrecht-Boston (2004).

[7] CElKO, V., GUldAN, M., MARCOKOVA, M.: On Jacobi Polynomials, Proc. of $5^{\text {th }}$ Workshop on Functional Analysis and its Applications in Mathematical Physics and Optimal Control, Nemecka 2005 (2005), 45-49.

[8] DIBLIK, J., KHUSAINOV, D.: Representation of Solutions of Discrete Delayed System $x(k+1)=A x(k)+B x(k-m)+f(k)$ with Commutative Matrices, J. Math. Anal. Appl. 318 (2006), 63-76.

[9] DIBLIK, J., KHUSAINOV, D., RUZICKOVA, M.: Controllability of Linear Discrete Systems with Constant Coefficients and Pure Delay, SIAM J. Control Optim, in press.

[10] DIBLIK, J., KUDELCIKOVA, M.: Inequalities for Positive Solutions of the Equation $\dot{y}(t)=-\left(a_{0}+a_{1} / t\right) x\left(t-\tau_{1}\right)-$ $-\left(b_{0}+b_{1} / t\right) x\left(t-\tau_{2}\right)$, Stud. Univ. Zilina Math. Ser. 17 (2003), no. 1, 27-46.

[11] DIBLIK, J., KUDELCIKOVA, M.: Inequalities for the Positive Solutions of the Equation $\dot{y}(t)=-\sum_{i=1}^{n}\left(a_{i}+b_{i} / t\right) y\left(t-\tau_{2}\right)$, Differential and Difference Equations and Applications, Hindawi Publ. Corp., New York, 2006, 341-350.

[12] DIBLIK, J., KUDELCIKOVA, M.: Nonoscillating Solutions of the Equation $x(t)=-(a+b / t) x(t-\tau)$, Stud. Univ. Zilina Math. Ser. 15 (2002), no. 1, 11-24.

[13] DIBLIK, J., KUDELCIKOVA, M.: Two Classes of Asymptotically Non-comparable Positive Solutions of the Equation $\dot{y}(t)=-f\left(t, y_{t}\right)$, (Submitted.)

[14] DIBLIK, J., RUZICKOVA, I., RUZICKOVA, M.: A General Version of the Retract Method for Discrete Equations, Acta Math. Sin. 23 (2007), no. 2, 341-348.

[15] DIBLIK, J., RUZICKOVA, M.: Asymptotic Behavior of Solutions and Positive Solutions of Differential Delayed Equations, Funct. Differ. Equ. 14 (2007), no. 1, 83-105. 
[16] DIBLIK, J., RUZICKOVA, M.: Convergence of the Solutions of the Equationy $\dot{y}(t)=\beta(t)[y(t-\delta)-y(t-\tau)]$ in the Critical Case, J. Math. Anal. Appl. 331 (2007), 1361-1370.

[17] DIBLIK, J., RUZICKOVA, M.: Existence of Positive Solutions of a Singular Initial Problem for a Nonlinear System of Differential Equations, Rocky Mountain J. Math. 34 (2004), 923-944.

[18] DIBLIK, J., RUZICKOVA, M.: Exponential Solutions of Equation $\dot{y}(t)=\beta(t)[y(t-\delta)-y(t-\tau)]$, J. Math. Anal. Appl. 294 (2004), no. 1, 273-287.

[19] DIBLIK, J., RUZICKOVA, M.: Inequalities for Solutions of Singular Initial Problems for Caratheodory Systems via Wazewski's Principle, Nonlinear Anal., (2007), doi: 10.1016/j.na.2007.11.006, in press.

[20] DIBLIK, J., RUZICKOVA, M., VAClAVIKOVA, B.: A Retract Principle on a Time scale, Stud. Univ. Zilina Math. Ser. 18 (2004), no. $1,37-44$.

[21] DIBLIK, J., VACLAVIKOVA, B.: Bounded Solutions of Discrete Equations on Discrete Real Time Scales, Funct. Differ. Equ. 14 (2007), no. 1, 67-82.

[22] HANUSTIAKOVA, E., OLACH, R.: Asymptotic and Oscillatory Properties of Neutral Differential Systems, Stud. Univ. Zilina Math. Ser. 19 (2005), no. 1, 9-18.

[23] HANUSTIAKOVA, E., OLACH, R.: Nonoscillatory Bounded Solutions of Neutral Differential Systems, Nonlinear Anal., http://dx.doi.org / 10.1016/j.na.2007.01014, in press.

[24] HANUSTIAKOVA, E., OLACH, R.: On Dynamics of Certain Economic Models, Komunikacie 4 (2005), 37-40.

[25] HANUSTIAKOVA, E., OLACH, R., SVANOVA, M.: Existence of Positive Periodic Solutions of Functional Differential Equations, Tatra Mount. Math. Publ., in press.

[26] KOVACIK, O.: On the Lebesgue Spaces and Spaces of Bounded q-variation, Tatra Mount. Math. Publ. 34 (2006), 189-200.

[27] KOVACIK, O., RAKOSNIK, J.: On Spaces $L^{p(x)}$ and $W^{k, p(x)}$, Czechoslovak Math. Journal 41 (1991), 592-618.

[28] LANGEROVA, M.: Solvability Conditions of Boundary Value Problems for Second Order Nonlinear Differential Systems, Tatra Mount. Math. Publ, in press.

[29] LANGEROVA, M., SHOVKOPLYAS, T.: Conditions for the Existence of a Solution of a Noether Boundary-value Problem for a Second-order System, Neliniini Kolyvannya 9, No. 3 (2006), 368-375 (English translation in: Nonlinear Oscillations: www.springer.com).

[30] MARCOKOVA, M., STANCEK, V.: On Polynomials Orthogonal in a Finite (Symmetric) Interval, Acta Ma-thematica 9 (2006), 209-214.

[31] MIHALY, T., Oscillations of Three-dimensional Differential Systems of Neutral Type, Nonlinear Anal. 66 (2007), no. 9, 2053-2063.

[32] OLACH, R.: Oscillation and Nonosdilation of First Order Nonlinear Delay Differential Equations, Act. Math. Universitatis Ostraviensis 12 (2004), 41-47.

[33] OLACH, R., SVANOVA, M.: Asymptotic Properties of Nonlinear Delay Differential Equations, Inter. Conf. APLIMAT 2007, Bratislava, (2007), 257-263.

[34] TAYLOR, M. A., WINIGATE, B. A., VINCENT, R. E.: An Algorithm for Computing Fekete Points in the Triangle, Siam J. Numer. Anal. 38/5 (2000), 1707-1720.

[35] VACLAVIKOVA, B.: Bounded Solutions of Dynamic Equations on Combined Time Scales, Tatra Mount. Math. Publ., in press.

[36] ZITNAN, P.: A Stable Discrete Least-squares Method for Membrane Eigenvalue Problems, Computers Math. Applic. (Submitted.)

[37] ZITNAN, P.: Discrete Least-squares Method for the Poisson Problems on Standard 2D Domains (in preparation). 